\title{
Simultaneous Two-angle Axial Ratiometry for Fast Live and Long- term Three-dimensional Super-resolution Fluorescence Imaging
}

Wenjie Liu ${ }^{1}$, Cuifang Kuang ${ }^{1,2,3}$, Yifan Yuan ${ }^{1}$, Zhimin Zhang ${ }^{1}$, Youhua Chen ${ }^{1,4}$, Yubing Han ${ }^{1}$, Liang $X u^{1}$, Meng Zhang, Yu-Hui Zhang, Yingke Xu' ${ }^{6}$ and Xu Liu ${ }^{1,2,3^{*}}$

${ }^{1}$ State Key Laboratory of Modern Optical Instrumentation, College of Optical Science and Engineering, Zhejiang University, Hangzhou, Zhejiang 310027, China

${ }^{2}$ Ningbo Research Institute, Zhejiang University, Ningbo, Zhejiang 315100, China

${ }^{3}$ Collaborative Innovation Center of Extreme Optics, Shanxi University, Taiyuan, Shanxi 030006, China

${ }^{4}$ Shanxi Provincial Key Laboratory for Biomedical Imaging and Big Data, North University of China, Taiyuan, Shanxi 030051, China

${ }^{5}$ Britton Chance Center for Biomedical Photonics, Wuhan National Laboratory for Optoelectronics-Huazhong University of Science and Technology, Wuhan, Hubei 430074, China

${ }^{6}$ Key Laboratory for Biomedical Engineering of Ministry of Education, Department of Biomedical Engineering, Zhejiang University, Hangzhou 310027, China

Corresponding Author: C. K. (cfkuang@zju.edu.cn) and X. L. (liuxu@zju.edu.cn) 


\begin{tabular}{|c|c|}
\hline Supplementary Files & Title \\
\hline Figure S1 & Flowchart of STARII and STARII-SIM reconstructions \\
\hline Figure S2 & Schematics of STARII and STARII-SIM imaging modes \\
\hline Figure S3 & Theoretical analysis of the STARII axial resolution \\
\hline Figure S4 & Simulated comparison using STARII and MA-TIRFM \\
\hline Figure S5 & Theoretical analysis of the axial overlap problem \\
\hline Figure S6 & SIM resolution analysis using Fourier ring correlation \\
\hline Figure S7 & $\begin{array}{r}\text { Long-term membrane curvature dynamics imaging using } \\
\text { STARII }\end{array}$ \\
\hline Table S1 & $\begin{array}{r}\text { Comparison of different TIRFM-based methods of } \\
\text { improving the axial resolution }\end{array}$ \\
\hline Note S1 & $\begin{array}{r}\text { STARII theory } \\
\text { Note S2 }\end{array}$
\end{tabular}




\section{Figure S1 Flowchart of STARII and STARII-SIM reconstructions}

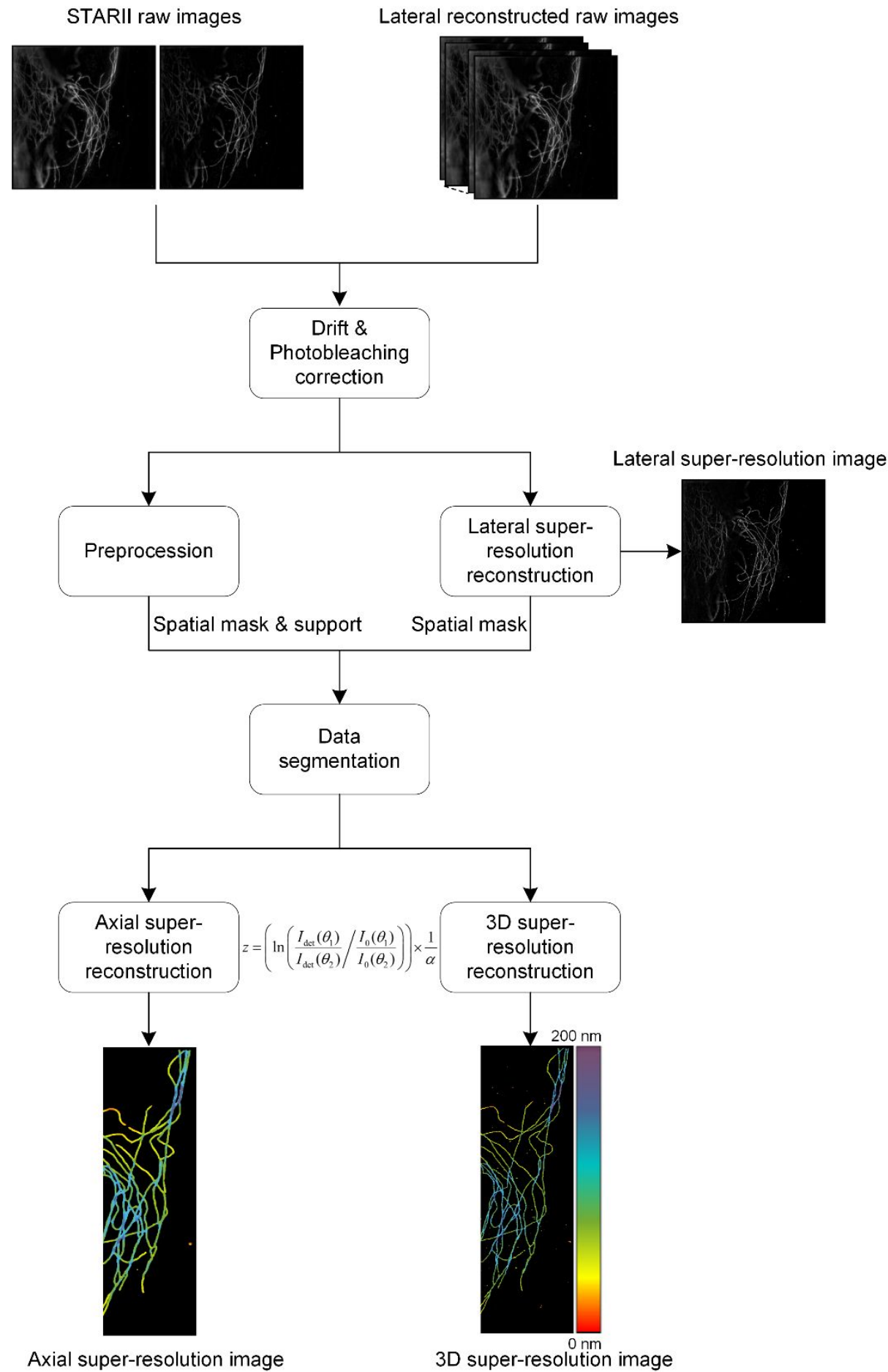

The reconstruction input for each frame is a raw image stack including two raw STARII images acquired at different TIRFM angles under azimuth-averaging illumination and a series of lateral reconstructed raw images acquired at the lower of the two incident angles used for STARII reconstruction (if necessary). The number of lateral reconstructed raw images depends on the method used. In the first optional step, lateral drift and photobleaching are corrected using corresponding ImageJ plugins. This step is needed especially in processed involving relatively large amounts of image data acquisition, such as long-term live-cell imaging. After that, 
STARII raw images are preprocessed with background subtraction. Lateral super-resolution reconstruction can also be performed with lateral reconstructed raw images. In this step, the same ROI can be selected to improve the reconstruction efficiency. Then, the preprocessed STARII raw images are segmented using Trainable Weka Segmentation to serve as their own spatial mask and spatial support for STARII reconstruction. For 3D superresolution STARII reconstruction, the lateral super-resolution image is used as a spatial mask to perform segmentation and abandon diffraction-limited frequencies in the raw STARII images without damaging the intensity relationship that is the basis for reconstruction. Finally, axial and 3D super-resolution reconstructions are performed using software written by the authors in MATLAB. The output is represented with color-coded depth. See Note S1 for additional theoretical details about STARII. 


\section{Figure S2 Schematics of four different imaging modes}

a
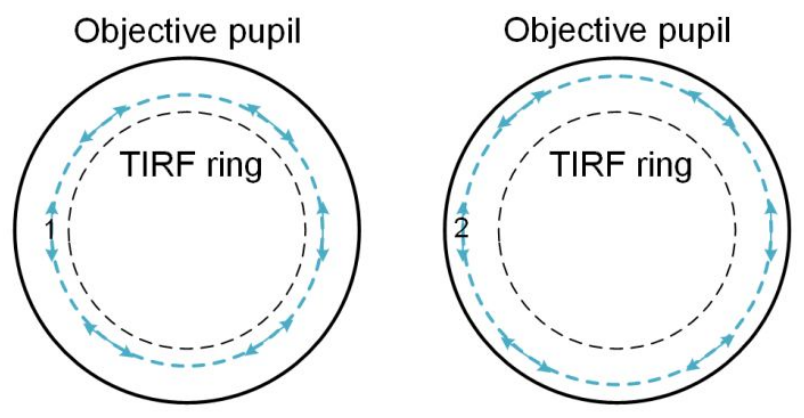

b
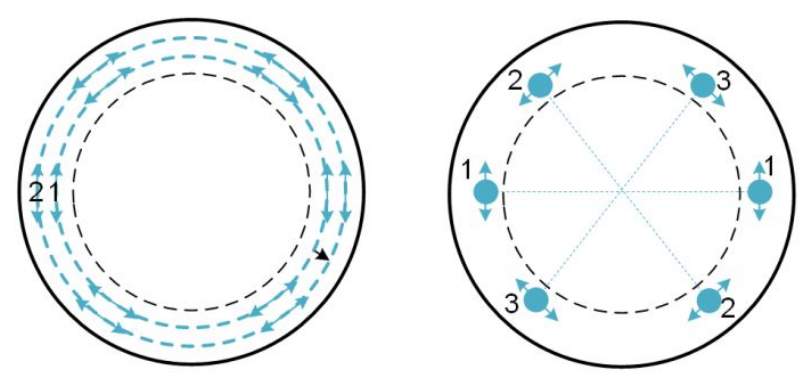

(a) In STARII imaging mode, two sequential s-polarized spinning-azimuth-TIRFM images at different incident angles need to be acquired. (b) In STARII-SIM imaging mode, two raw STARII images are obtained firstly. Then, nine raw TIRF-SIM images at different azimuths and different phases are acquired. Note that all of the lateral super-resolution raw images should be acquired at the smaller STARII acquisition incident angle to provide the lateral super-resolution information projected over the whole axial range that STARII then covers. 
Figure S3 Theoretical analysis of the STARII axial resolution

a

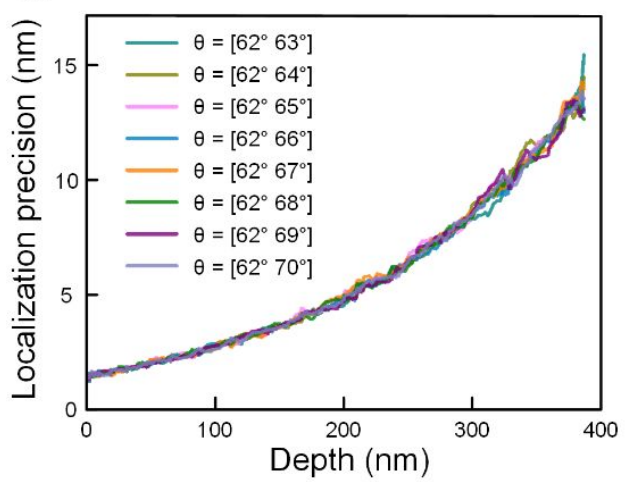

C

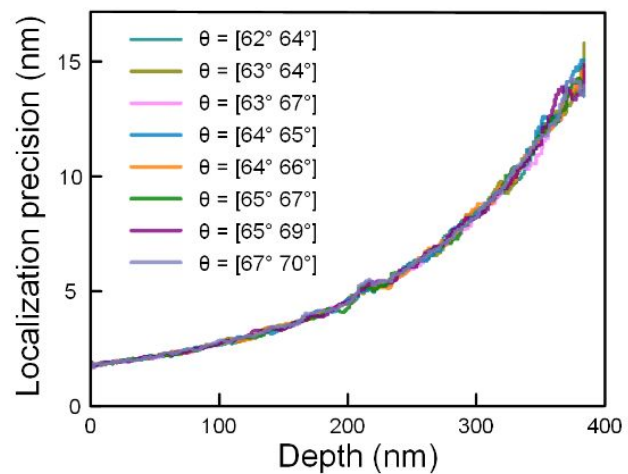

b

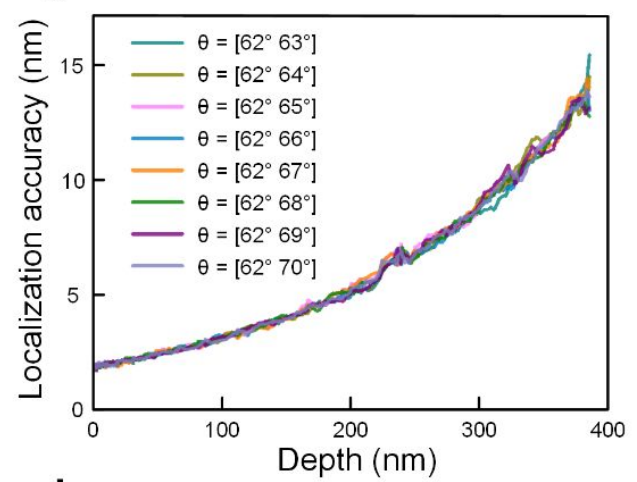

d

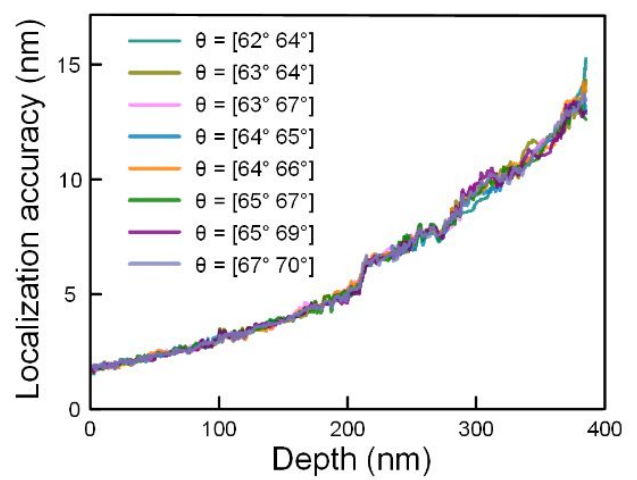

Theoretical axial localization precision (a, c) and localization accuracy (b, d) of STARII at different sample depths and incident angles. The simulation conditions used to obtain a-d were $\lambda=488 \mathrm{~nm}, \mathrm{SNR}=20, n_{1}=1.515$, and $n_{2}=$ 1.33 . 


\section{Figure S4 Simulated comparison using STARII and MA-TIRFM}
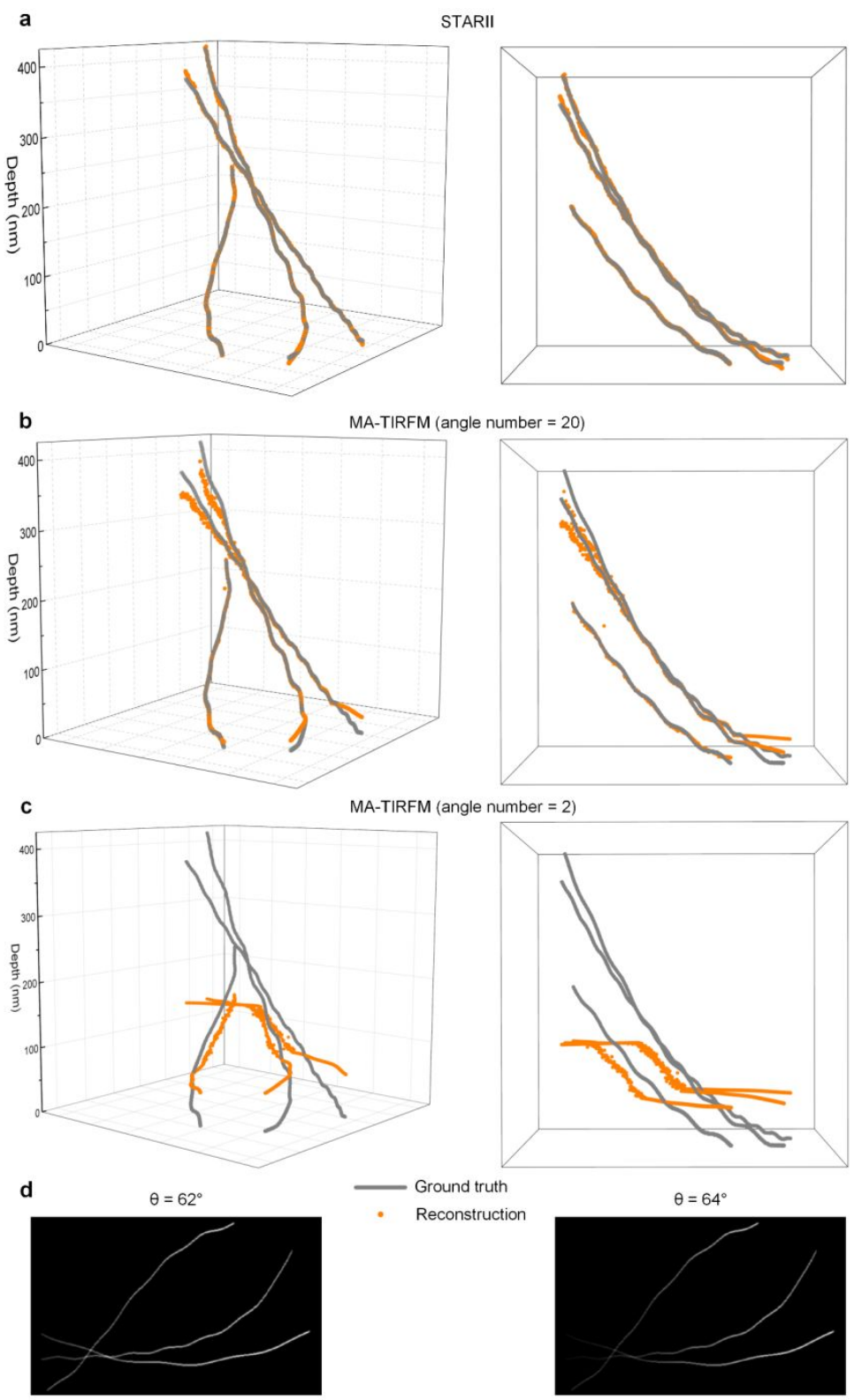

(a) Front view (left) and side view (right) of the STARII reconstruction results. The simulation conditions were $\lambda=$ $488 \mathrm{~nm}, \theta=\left[62^{\circ}, 64^{\circ}\right], \mathrm{SNR}=20, n_{1}=1.515$, and $n_{2}=1.33$. (b) Front view (left) and side view (right) of reconstruction results obtained using the alternating direction method of multipliers-based MA-TIRFM. The simulation conditions were $\lambda=488 \mathrm{~nm}, \theta=\left[61.5^{\circ}: 0.5^{\circ}: 71^{\circ}\right], \mathrm{SNR}=20, n_{1}=1.515$, and $n_{2}=1.33$. (c) Front view (left) and side view (right) of reconstruction result using the alternating direction method of multipliers-based MA-TIRFM. The simulation conditions were $\lambda=488 \mathrm{~nm}, \theta=\left[62^{\circ}, 64^{\circ}\right]$, SNR $=20, n_{1}=1.515$, and $n_{2}=1.33$. (d) Two raw TIRFM images used for STARII reconstruction. Left: $\theta=62^{\circ}$; right: $\theta=64^{\circ}$. The simulation conditions were the same as those used to obtain a. 


\section{Figure S5 Theoretical analysis of the axial overlap problem}
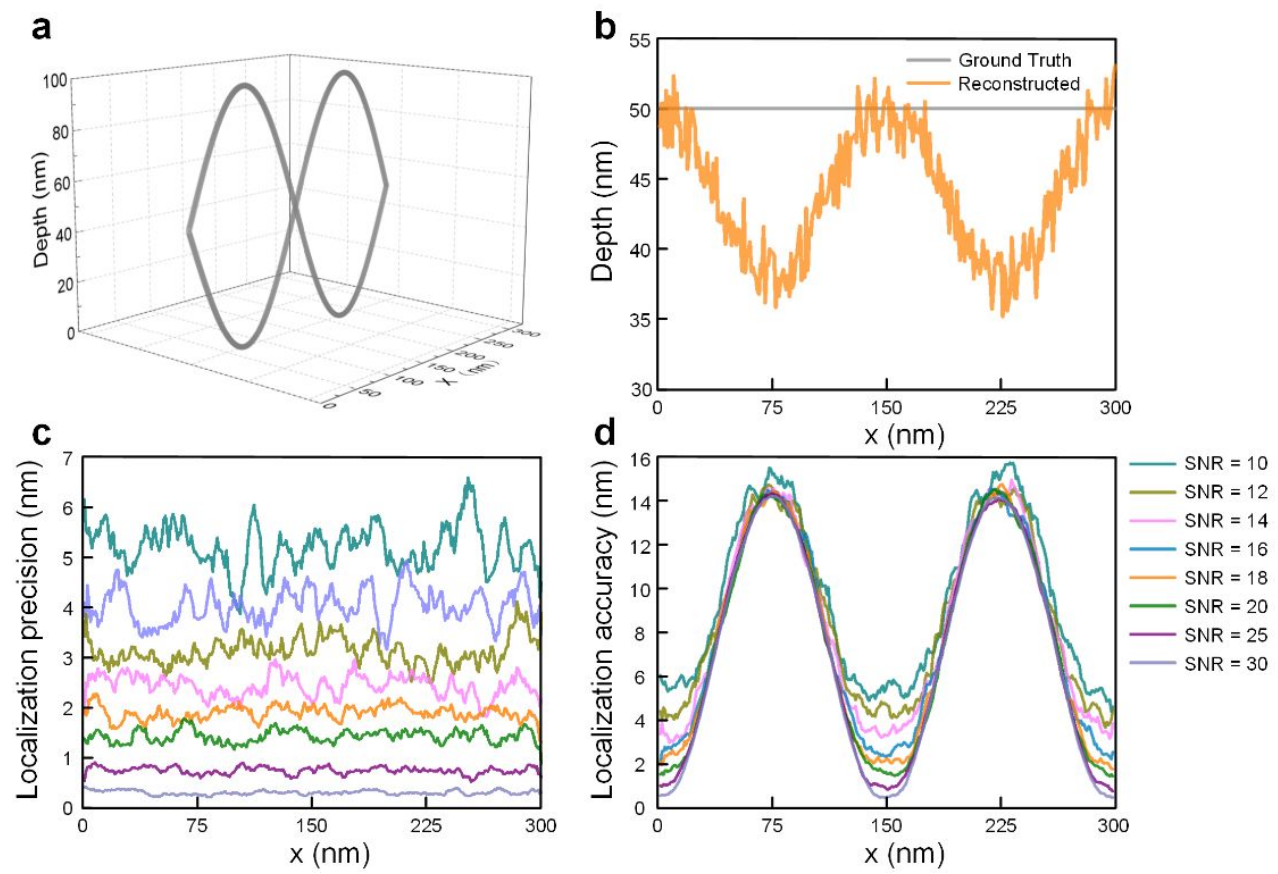

(a) 3D visualization of the ground-truth microtubule sample overlapped axially. The axial separation ranges from 0 $\mathrm{nm}$ to $100 \mathrm{~nm}$ at a lateral distance of $75 \mathrm{~nm}$. (b) Reconstructed depth error at different $\mathrm{x}$ positions corresponding to different axial separation. The gray line represents the average axial position of the axial overlap points. The orange line represents the reconstructed depth at the corresponding point. The simulation conditions were $\lambda=488 \mathrm{~nm}, \theta=$ $\left[62^{\circ}, 64^{\circ}\right], \mathrm{SNR}=20, n_{1}=1.515$, and $n_{2}=1.33$. (c, d) Theoretical axial localization precision (c) and localization accuracy (d) of STARII with different axial separations and SNRs. The localization precision and accuracy at each lateral position were calculated based on the average axial positions of the two axial overlap points. The simulation conditions used to obtain (c, d) were $\lambda=488 \mathrm{~nm}, \theta=\left[62^{\circ}, 64^{\circ}\right], n_{1}=1.515$, and $n_{2}=1.33$. 
Figure S6 SIM resolution analysis using Fourier ring correlation
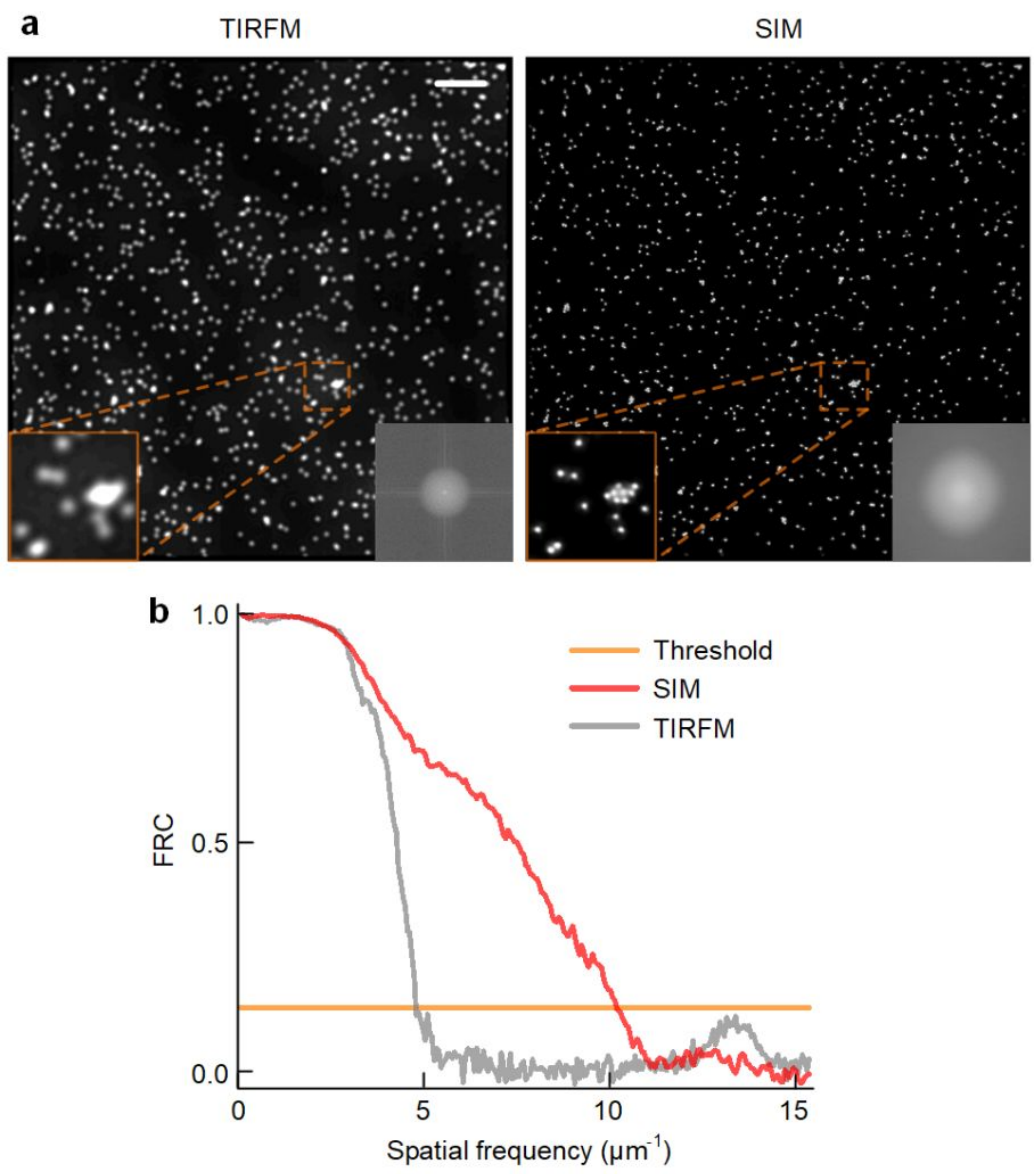

(a) TIRFM (left) and SIM (right) images of $60 \mathrm{~nm}$ fluorescent nanoparticles (Invitrogen, 505/515). The bottom-left images are the corresponding magnified images of the orange boxed ROI, illustrating the resolution improvement in the spatial domain. The bottom-right images show the frequency distributions of the corresponding whole images, illustrating the resolution improvement in the frequency domain. (b) Fourier ring correlation (FRC) curve calculated using the experimental results in a. The 1/7 fixed threshold was chosen to define the effective cut-off frequencies, showing the TIRFM and SIM resolutions of $\sim 205$ $\mathrm{nm}$ and $\sim 97 \mathrm{~nm}$, respectively. Scale bar, $3 \mu \mathrm{m}$ in (a). 
Figure S7 Long-term membrane curvature dynamics imaging using STARII
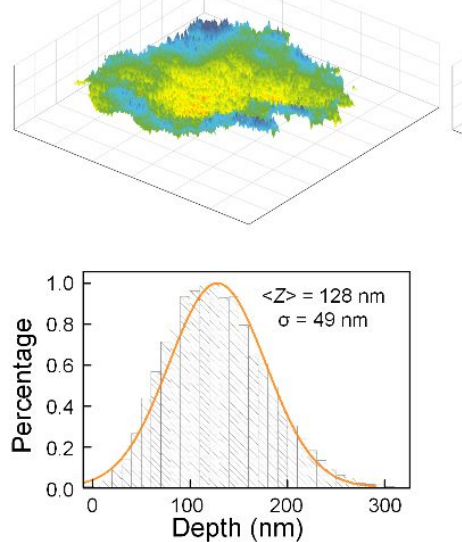

d
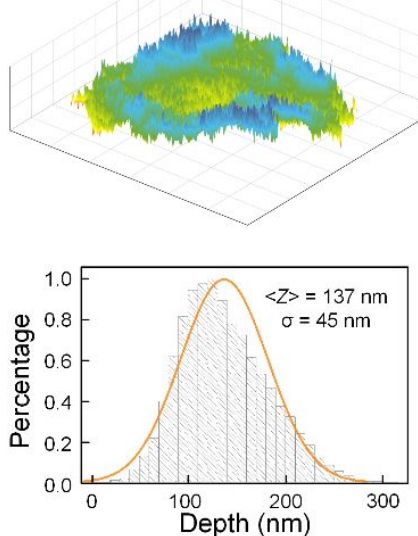

g
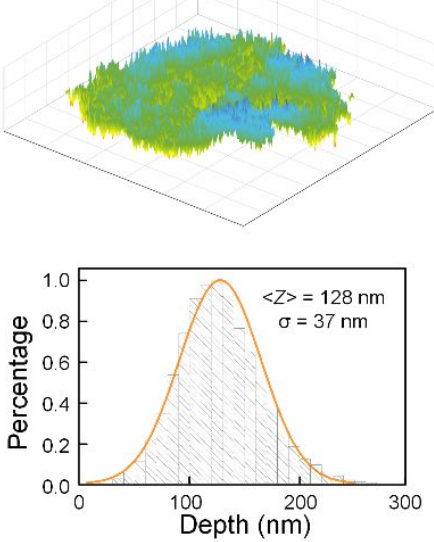

b
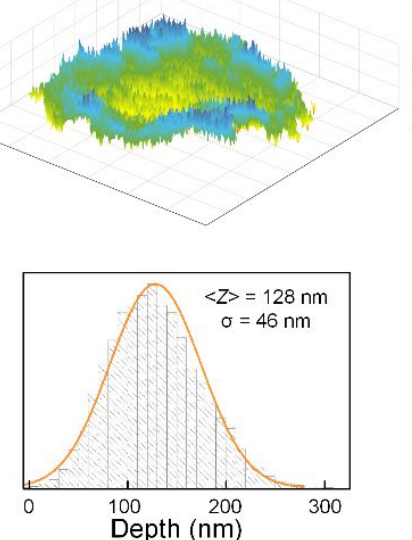

e

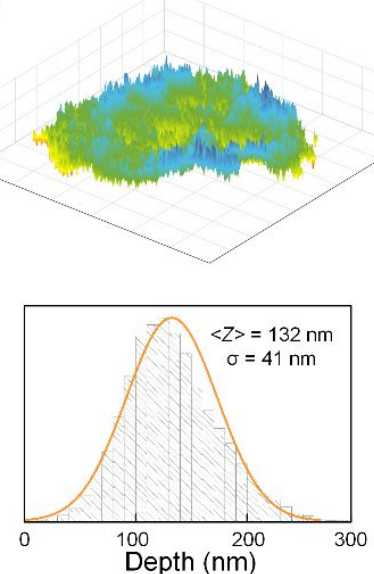

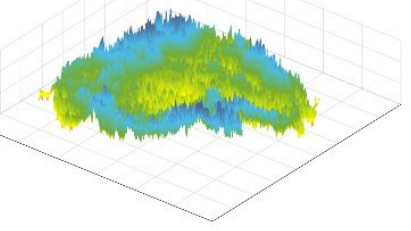

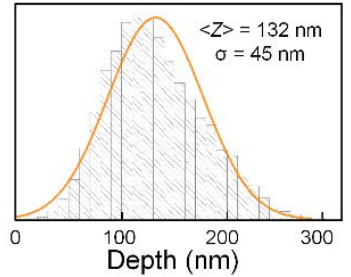

f
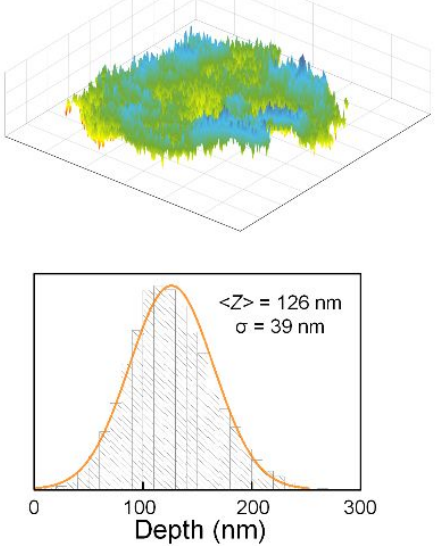

$\mathbf{h}$
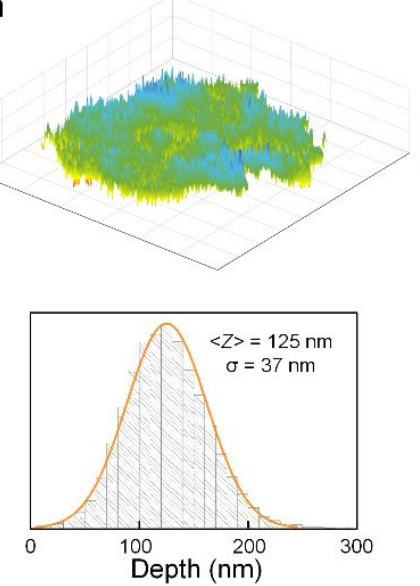

i

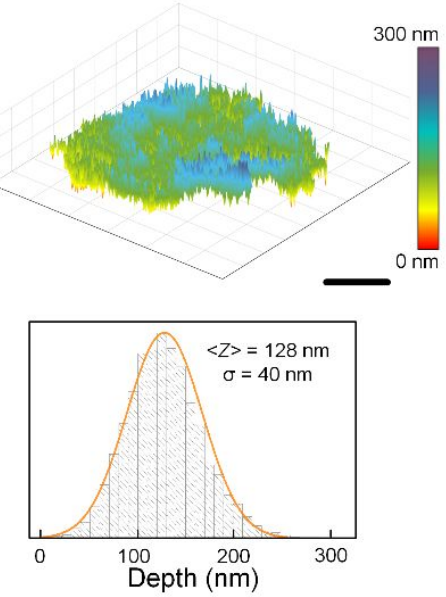

(a-i) Morphology evolution (top) and corresponding normalized depth histograms (bottom) of the cell plasma membrane with a time interval of $5 \mathrm{~min}$. The solid orange curves show the Gaussian fits to the experimental data. Scale bar, $3 \mu \mathrm{m}$ in (a-i). 
Table S1 Comparison of different TIRFM-based methods of improving the axial resolution

\begin{tabular}{|c|c|c|c|}
\hline Method & Principle & $\begin{array}{c}\text { Axial } \\
\text { resolution }(\mathrm{nm})\end{array}$ & Limitation $^{\text {b) }}$ \\
\hline $\mathrm{TIRFM}^{[1,2]}$ & $\begin{array}{l}\text { When the light is incident from a high- } \\
\text { refractive-index medium into a low- } \\
\text { refractive-index medium at an angle greater } \\
\text { than the critical angle, an evanescent wave is } \\
\text { produced that illuminates the sample on an } \\
\text { axial spatial scale down to a few hundred } \\
\text { nanometers with a high signal-to-background } \\
\text { ratio. }\end{array}$ & $\begin{array}{l}\text { On the order of } \\
\text { the wavelength }\end{array}$ & / \\
\hline $\begin{array}{c}\text { MA- } \\
\text { TIRFM[3] }^{[3]}\end{array}$ & $\begin{array}{l}\text { Two properties of the evanescent wave can be } \\
\text { used to improve the axial resolution of } \\
\text { TIRFM further and to determine the emitter } \\
\text { depth: 1. the exponential intensity decay with } \\
\text { increasing depth relative to the coverslip and } \\
\text { 2. the decrease in penetration depth with } \\
\text { increasing incident angle. MA-TIRFM } \\
\text { reconstruction can be performed via the } \\
\text { following seven methods: }\end{array}$ & I & / \\
\hline $\begin{array}{c}\text { Inverse } \\
\text { problem } \\
\text { solving[4-7] }\end{array}$ & $\begin{array}{l}\text { This approach firstly involves building the } \\
\text { relationship among the excitation field, } \\
\text { sample spatial distribution, and acquired MA- } \\
\text { TIRFM images. The relationship is then } \\
\text { treated as an inverse problem that can be } \\
\text { solved with many convex optimization } \\
\text { algorithms. }\end{array}$ & $\sim 40-50$ & $\begin{array}{l}\text { Tens of raw images } \\
\text { needed. }\end{array}$ \\
\hline $\begin{array}{l}\text { Curve fitting } \\
\text { type- }{ }^{[8]}\end{array}$ & $\begin{array}{l}\text { This approach deduces a linear relationship } \\
\text { between the axial emitter position and the } \\
\text { fluorescence intensity of each MA-TIRFM } \\
\text { image pixel. With this linear relationship, the } \\
\text { axial position can then be fitted pixel by } \\
\text { pixel. }\end{array}$ & $\sim 10-20^{\mathrm{a})}$ & $\begin{array}{l}\text { Tens of raw images } \\
\text { needed. }\end{array}$ \\
\hline $\begin{array}{l}\text { Curve fitting } \\
\text { type-II }{ }^{[9]}\end{array}$ & $\begin{array}{l}\text { This approach involves determining the axial } \\
\text { position of each pixel by minimizing the } \\
\text { difference between the experimental and } \\
\text { theoretical values of the excitation intensity at } \\
\text { the interface. }\end{array}$ & $\sim 40^{\mathrm{a})}$ & $\begin{array}{l}\text { Tens of raw images } \\
\text { needed. }\end{array}$ \\
\hline
\end{tabular}




\begin{tabular}{|c|c|c|c|}
\hline $\begin{array}{c}\text { Sequential } \\
\text { imaging and } \\
\text { bleaching }{ }^{[10]}\end{array}$ & $\begin{array}{l}\text { This approach involves resolving diffraction- } \\
\text { limited structures in the axial direction by } \\
\text { sequential bleaching and imaging of the } \\
\text { sample layer by layer. }\end{array}$ & $\sim 20$ & $\begin{array}{l}\text { 1. Tens of raw } \\
\text { images needed; } \\
\text { 2. High illumination } \\
\text { intensity needed. }\end{array}$ \\
\hline $\begin{array}{l}\text { Differential } \\
\text { evanescence } \\
\text { nanometry[11] }\end{array}$ & $\begin{array}{l}\text { By tagging different axial positions in a } \\
\text { sample with different fluorescent dyes, the } \\
\text { ratio between two wide-field images and two } \\
\text { TIRFM images is used to measure the } \\
\text { separation between the two fluorophores. }\end{array}$ & $\sim 10^{\mathrm{a})}$ & $\begin{array}{l}\text { 1. Four raw images } \\
\text { needed; } \\
\text { 2. Relative depth } \\
\text { measurement; } \\
\text { 3. Dual-color dyes } \\
\text { labeling. }\end{array}$ \\
\hline $\begin{array}{l}\text { Normalized } \\
\text { TIRFM }^{[12,13]}\end{array}$ & $\begin{array}{l}\text { This approach involves determining the axial } \\
\text { position of each pixel by normalizing TIRFM } \\
\text { images with corresponding epi-fluorescence } \\
\text { images. }\end{array}$ & $\sim 10-20^{\mathrm{a})}$ & $\begin{array}{l}\text { Only suitable for } \\
\text { samples with known } \\
\text { shape and size such } \\
\text { as vesicles. }\end{array}$ \\
\hline $\begin{array}{l}\text { Simultaneous } \\
\text { two- } \\
\text { wavelength } \\
\quad \text { axial } \\
\text { ratiometry }{ }^{[14]}\end{array}$ & $\begin{array}{l}\text { Unlike differential evanescence nanometry, } \\
\text { this approach involves tagging the same } \\
\text { position in a sample with two different } \\
\text { fluorescent dyes and then using the ratio } \\
\text { between two wide-field images and two } \\
\text { TIRFM images to measure the relative axial } \\
\text { positions of proteins. }\end{array}$ & $\sim 20^{\mathrm{a})}$ & $\begin{array}{l}\text { 1. Four raw images } \\
\text { needed; } \\
\text { 2. Relative depth } \\
\text { measurement; } \\
\text { 3. Dual-color dyes } \\
\text { labeling. }\end{array}$ \\
\hline $\begin{array}{l}\text { Supercritical } \\
\text { angle } \\
\text { fluorescence } \\
\text { microscopy }{ }^{[15} \\
-17]\end{array}$ & $\begin{array}{l}\text { The emitted fluorescence can be divided into } \\
\text { undercritical- and supercritical-angle } \\
\text { fluorescence signals according to the critical } \\
\text { angle. Like the evanescent wave, the } \\
\text { supercritical angle fluorescence signal decays } \\
\text { exponentially along the axial direction. The } \\
\text { ratio between these two fluorescence signals } \\
\text { can be used to determine the axial position of } \\
\text { an emitter. }\end{array}$ & $\sim 15^{\text {a) }}$ & I \\
\hline
\end{tabular}

a)These axial resolutions were defined as the axial localization precisions.

b) The common limitation of TIRFM-based approaches and supercritical angle fluorescence microscopy is their poor imaging depth which is determined by the evanescent wave theory. 


\section{Note S1 STARII theory}

To express the intensity of the evanescent wave, we first define a coordinate system in which the incident plane is the $x$-z plane, with $x$ parallel to the interface and $\mathrm{z}$ perpendicular to it. The $y$-axis is normal to the incident plane. Then, according to electromagnetic theory, the complex amplitude of the evanescent wave can be written as

$$
E=E_{0} e^{-k z \sqrt{n_{1}^{2} \sin ^{2} \theta-n_{2}^{2}}}
$$

where $E_{0}$ and $k$ are the amplitude and wave vector of the evanescent wave, respectively; $\theta \square$ is the incident angle; and $n_{1}$ and $n_{2}$ are the refractive index of the incident medium and the refracted medium, respectively. Equation S1 shows that the amplitude of the evanescent wave decays exponentially with the depth $z$ relative to the coverslip. When the decayed amplitude is equal to $1 / \mathrm{e}$ of the amplitude at the interface $(z=0)$, the depth is defined as the penetration depth $d$ and can be deduced as

$$
d=\frac{\lambda}{4 \pi \sqrt{n_{1}^{2} \sin ^{2} \theta-n_{2}^{2}}}
$$

where $\lambda$ is the wavelength of the incident light. Thus, the intensity of the evanescent excitation field $I_{\text {exc }}$ in TIRFM can be written as

$$
I_{e x c}=I_{0} e^{\frac{-z}{d}}
$$

where $I_{0}$ is the light intensity at the interface, which is a function of the incident angle and incident light polarization ${ }^{[1]}$. For s-polarized incident light in this study, the evanescent field is also spolarization. $I_{0}$ can be written as

$$
I_{0}=\chi_{s} \frac{4 \cos ^{2} \theta}{1-n^{2}}
$$

where $\chi_{s}$ is the polarized intensity of the incident electric field in the glass. Note that incident light with other polarizations, such as $\mathrm{p}$ and circular polarization, can also be used if the s-polarized expression of $I_{0}$ in the reconstruction algorithm is replaced with that for the corresponding polarization. Considering the azimuthal averaging, the acquired single-angle TIRFM image can be described by 
where $\varphi$ is the azimuthal angle; $\varepsilon(\varphi)$ is the factor that takes into account the influence of the illumination direction on the excitation field; $\phi(z)$ represents the local distribution of the molecules; $\gamma(z)$ is the factor associated with the absorption rate, dipole orientation, and quantum field of the molecule dyes; and $\eta(z)$ is the detection efficiency of the system. From Equation S5, we can see that the intensity distribution of a single-angle TIRFM image is determined not only by the evanescent excitation field, but also by many unknown parameters mentioned above, which makes it difficult to analyze TIRFM images quantitatively. However, thanks to the incident angle-independent nature of these parameters, this problem can be solved by analyzing multiple TIRFM images recorded at different incident angles. Therefore, we propose a new factor $\sigma$ to represent these parameters:

$\sigma(z)=\left(\int \varepsilon(\varphi) d \varphi\right) \phi(z) \gamma(z) \eta(z)$

By plugging Equation S3 and S6 into S5, we can obtain

$$
I_{\mathrm{det}}=\int \sigma(z) I_{0} e^{\frac{-z}{d}} d z
$$

STARII assumes all fluorophores to be at a position $z$, i.e., no axial overlapping occurs, hence Equation S7 can be finally simplified to

$$
I_{\mathrm{det}}=\sigma I_{0} e^{\frac{-z}{d}}
$$

Note that although Equation S8 and S3 are very similar in form, their meanings are completely different.

Therefore, the TIRFM images acquired at two incident angles $\theta_{1}$ and $\theta_{2}$ are

$$
\begin{aligned}
& I_{\text {det }}\left(\theta_{1}\right)=\sigma I_{0}\left(\theta_{1}\right) e^{\frac{-z}{d\left(\theta_{1}\right)}} \\
& I_{\operatorname{det}}\left(\theta_{2}\right)=\sigma I_{0}\left(\theta_{2}\right) e^{\frac{-z}{d\left(\theta_{2}\right)}}
\end{aligned}
$$

The influence of $\sigma$ can be eliminated by dividing two detection intensities

$$
\frac{I_{\mathrm{det}}\left(\theta_{1}\right)}{I_{\mathrm{det}}\left(\theta_{2}\right)}=\frac{I_{0}\left(\theta_{1}\right)}{I_{0}\left(\theta_{2}\right)} e^{z \alpha}
$$


where

$\alpha=\frac{d\left(\theta_{1}\right)-d\left(\theta_{2}\right)}{d\left(\theta_{1}\right) d\left(\theta_{2}\right)}$

For simplicity, taking the logarithm of both sides of Equation S11, we have

$\ln \left(\frac{I_{\mathrm{det}}\left(\theta_{1}\right)}{I_{\mathrm{det}}\left(\theta_{2}\right)}\right)=\ln \left(\frac{I_{0}\left(\theta_{1}\right)}{I_{0}\left(\theta_{2}\right)}\right)+z \alpha$

Solving for $z$ gives

$z=\left(\ln \left(\frac{I_{\operatorname{det}}\left(\theta_{1}\right)}{I_{\operatorname{det}}\left(\theta_{2}\right)} / \frac{I_{0}\left(\theta_{1}\right)}{I_{0}\left(\theta_{2}\right)}\right)\right) \times \frac{1}{\alpha}$

The variables in Equation S14 can all be calculated or detected directly. Thus, the absolute depth of the fluorophore relative to the coverslip can be obtained using two TIRFM images acquired at different incident angles. 


\section{Note S2 Materials and methods}

Experimental setup. All of the experiments were performed using our previously reported home-built MAIM system, ${ }^{[6]}$ although STARII imaging can actually be performed using a conventional TIRFM system.

When measuring the excitation laser power at the output of the objective lens using a silicon photodiode power probe (Thorlabs, S170C), the average laser intensity ranged from $0.7 \mathrm{~W} / \mathrm{cm}^{2}$ to $10 \mathrm{~W} / \mathrm{cm}^{2}$ depending on the sample and imaging method. The typical exposure time of the EMCCD was $10-50 \mathrm{~ms}$, implying an acquisition rate of $>11$ fps in STARII imaging and 2 fps in STARIISIM imaging for a field of view of $512 \times 512$ pixels, which can easily be improved by reducing the exposure time and image size or using a faster sCMOS camera.

\section{Sample preparation.}

Fluorescent nanoparticle preparation. The fluorescent nanoparticles $(60 \mathrm{~nm}$, Invitrogen) used to obtain Figure S10 were firstly diluted in absolute ethyl alcohol at a concentration of 1:800 and then vibrated for 20 min using ultrasonic washer. To clean the coverslips and weaken the surface tension, we immersed them in poly-L-lysine (Sigma) for $10 \mathrm{~min}$. Then, the particle solution was dropped onto the coverslips using capillaries. After natural spreading, we used Mowiol mounting medium to mount the coverslips on microscope slides.

Fixed-cell preparation. U2OS cells were cultured in McCoy's 5A medium (Thermo Fisher Scientific, Inc.). All of the media were supplemented with $10 \%(\mathrm{v} / \mathrm{v})$ fetal bovine serum (Thermo Fisher Scientific, Inc.), and the cultures were maintained at $37{ }^{\circ} \mathrm{C}$ in a humidified $5 \% \mathrm{CO}_{2}$ environment. U2OS cells were seeded in Nunc Glass Bottom Dishes ( $\Phi 12 \mathrm{~mm}$, Thermo Fisher Scientific, Inc.) at a density of $1.5-2.0 \times 10^{4}$ per well in growth medium $(150 \mu \mathrm{L})$. After overnight incubation, the cells were washed three times with phosphate buffered saline. For the microtubule labeling used in Figure 
2d-f, the U2OS cells were incubated with SiR-tubulin (Cytoskeleton, Inc.) at a concentration of $1 \mu \mathrm{M}$ in a growth medium at $37^{\circ} \mathrm{C}$ for $30-60$ min and washed three times with the growth medium before imaging.

Live-cell preparation. For the ER labeling used in Figure 3, U2OS cells were transfected with SNAPSec61 $\beta$ using Lipofectamine 3000 (Thermo Fisher Scientific, Inc.) and cultured in an incubator at 37 ${ }^{\circ} \mathrm{C}$ with $5 \% \mathrm{CO}_{2}$ for an additional $24 \mathrm{~h}$ before the experiments. After that, the cells were incubated with SiR-SNAP (New England Biolabs, Inc.) at a concentration of $1 \mu \mathrm{M}$ in a growth medium at $37{ }^{\circ} \mathrm{C}$ for 30-60 min, and washed three times with the growth medium before imaging.

For the cell plasma membrane labeling used in Figures 4 and S7, the cells were incubated with DiD (5 $\mu \mathrm{M}$; Yeasen Biological Technology Co., Ltd., Shanghai, China) in a $5 \% \mathrm{CO}_{2}$ atmosphere at $37{ }^{\circ} \mathrm{C}$ for $20 \mathrm{~min}$. Then, the supernatant was discarded, and the cells were post-incubated with a growth medium in a $5 \% \mathrm{CO}_{2}$ atmosphere at $37{ }^{\circ} \mathrm{C}$ for $10 \mathrm{~min}$. The cells were washed twice gently with phosphate buffered saline and immersed in phenol red-free Dulbecco's Modified Eagle's Medium (Thermo Fisher Scientific, Inc.) prior to optical imaging.

Theoretical analysis. All of the STARII simulations were constructed in MATLAB with the parameters set to match the practical experimental conditions: $\lambda=488 \mathrm{~nm}, n_{1}=1.515$, and $n_{2}=1.33$. The other parameters, including the incident angle $\theta$ and SNR, were changed in different simulations as indicated in the main text. The microtubule simulations involved three main steps. First, a 3D microtubule sample was generated using exponential and trigonometric functions to depict the 3D growth tendencies and local bending of the tubules, respectively. Then, two raw TIRFM images were produced considering the characteristics of the corresponding evanescent wave illumination fields (see Note S1 for further details). Each TIRFM image was the 2D intensity projection of the whole illuminated 3D sample volume. At this stage, Gaussian white noise with different standard deviations was added to evaluate the influence of noise on the STARII measurement. Finally, the axial position 
was calculated pixel by pixel using Equation 2. The axial localization precision (defined as the axial resolution in this paper) and localization accuracy were determined by performing 1000 measurements under the same conditions.

Imaging modes and image acquisition. Two imaging modes, STARII and STARII-SIM, were used in this study (Figure S2). In STARII-SIM mode, the lateral super-resolution raw SIM data and axial super-resolution raw STARII data were recorded subsequently. All of the data sets were acquired using the MAIM system and the corresponding control software written on the LabVIEW platform.

Drift correction. Considering that the axial positions in the STARII-like methods were all calculated pixel by pixel, drift correction (pixel alignment) between sequential TIRFM images acquired at different incident angles was necessary for depth reconstruction. Lateral drift correction was conducted after acquiring a whole stack of raw images. An extended Marquardt-Levenberg optimization algorithm (“StackReg” ImageJ plugin) was used to correct lateral drift."18] For axial drift correction, a near infrared light emission diode was focused off-axis onto the back focal plane of the objective. The corresponding total internal reflection light beam from the coverslip-sample interface was detected by a line-array charge coupled device. The center of the detected light in the image was compared with the calibrated value to measure the axial position of the sample. If the light was defocused, a feedback loop system corrected the axial drift in real time.

Photobleaching correction. Photobleaching is the light-induced irreversible destruction of fluorescent dyes that causes the SNR to decrease and thus should be prevented in most biological imaging experiments, especially in time-lapse live-cell studies conducted over long time periods. In general, the factors affecting photobleaching can be divided into two categories: chemical-propertyrelated and experimental-condition-related factors. The chemical-property-related factors, such as the quantum yield, extinction coefficient, and photostability of the fluorophores, are closely associated 
with the properties of the fluorophore themselves and the labeling strategy. In some cases, photobleaching can be further prevented by reducing the amount of oxygen in the medium at the cost of phototoxicity to live cells. Considering the experimental conditions, photobleaching is best relieved by optimizing the microscope system to suppress noise and maximize the signal so that the minimal possible light dose can be used. More specifically, to minimize time wastage and bleaching, the illumination intensity should be kept low when searching for a suitable imaging cell or when focusing the image plane on the camera and then be increased to the proper level to acquire raw images. Moreover, we performed standard photobleaching correction using the EMBL "Bleach Correction" plugin in Image.$^{[19]}$

Data segmentation. To eliminate the effects of the background, which may introduce fake depth information in all STARII-like reconstruction methods, it is necessary to subtract the background and segment interesting structures from the raw image data. ${ }^{[6,8,14,17,20-22]}$ In this study, we performed data segmentation using a robust machine-learning-based tool (the Trainable Weka Segmentation plugin in ImageJ).[23]

Data processing. Several different data sets were acquired during the study, typically a stack of two images for each STARII frame and eleven images for each STARII-SIM frame. The main purpose of the processing routines in each frame was to map the sample depth relative to the coverslip with the highest possible localization precision and accuracy. In general, the processing for generating a final 3D super-resolution image was accomplished in six steps: drift correction, photobleaching correction, lateral super-resolution reconstruction, data segmentation, STARII depth reconstruction, and image display (Figure S1). The drift correction, photobleaching correction, and data segmentation procedures based on ImageJ software are explained above. The SIM and STARII reconstruction algorithms were all self-written in MATLAB. Color-coded depth and corresponding 3D visualization images (except those shown in Figure 2h, i) were displayed with customized color bars using MATLAB. The 3D 
visualizations in Figure 2h, i, quantitative resolution representations and depth counts were obtained using Origin software. 
Supplementary References:

(1) Axelrod, D. Total internal reflection fluorescence microscopy in cell biology. Traffic 2001, 2, 764-774.

(2) Steyer, J. A.; Almers, W. A real-time view of life within $100 \mathrm{~nm}$ of the plasma membrane. Nat. Rev. Mol. Cell Bio. 2001, 2, 268-276.

(3) Liu, W.; Toussaint, Jr. K.; Okoro, C.; Zhu, D.; Chen, Y.; Kuang, C.; Liu, X. Breaking the axial diffraction limit: a guide to axial super-resolution fluorescence microscopy. Laser \& Photonics Rev. 2018, 12, 1700333.

(4) Olveczky, B. P.; Periasamy, N.; Verkman, A. Mapping fluorophore distributions in three dimensions by quantitative multiple angle-total internal reflection fluorescence microscopy. Biophy. J. 1997, 73, 2836-2847.

(5) Yang, Q.; Karpikov, A.; Toomre, D.; Duncan, J. S. 3-D reconstruction of microtubules from multi-angle total internal reflection fluorescence microscopy using Bayesian framework. IEEE Trans. Image Process. 2011, 20, 2248-2259.

(6) Chen, Y.; Liu, W.; Zhang, Z.; Zheng, C.; Huang, Y.; Cao, R.; Zhu, D.; Xu, L.; Zhang, M.; Zhang, Y.-H.; Fan, J.; Jin, L.; Xu, Y.; Kuang, C.; Liu, X. Multi-color live-cell superresolution volume imaging with multi-angle interference microscopy. Nat. Commun. 2018, 9, 4818.

(7) Boulanger, J.; Gueudry, C.; Münch, D.; Cinquin, B.; Paul-Gilloteaux, P.; Bardin, S.; Guérin, C.; Senger, F.; Blanchoin, L.; Salamero, J. Fast high-resolution 3D total internal reflection fluorescence microscopy by incidence angle scanning and azimuthal averaging. Proc. Natl. Acad. Sci. USA 2014, 111, 17164-17169.

(8) Dos Santos, M. C.; Déturche, R.; Vézy, C.; Jaffiol, R. Topography of cells revealed by variable-angle total internal reflection fluorescence microscopy. Biophy. J. 2016, 111, 13161327. 
(9) Jin, L.; Wu, J.; Xiu, P.; Fan, J.; Hu, M.; Kuang, C.; Xu, Y.; Zheng, X.; Liu, X. Highresolution $3 \mathrm{D}$ reconstruction of microtubule structures by quantitative multi-angle total internal reflection fluorescence microscopy. Opt. Commun. 2017, 395, 16-23.

(10) Fu, Y.; Winter, P. W.; Rojas, R.; Wang, V.; McAuliffe, M.; Patterson, G. H. Axial superresolution via multiangle TIRF microscopy with sequential imaging and photobleaching. Proc. Natl. Acad. Sci. USA 2016, 113, 4368-4373.

(11) Saffarian, S.; Kirchhausen, T. Differential evanescence nanometry: live-cell fluorescence measurements with $10-\mathrm{nm}$ axial resolution on the plasma membrane. Biophy. J. 2008, 94, $2333-2342$.

(12) Dos Santos, M. C.; Vézy, C.; Jaffiol, R. Nanoscale characterization of vesicle adhesion by normalized total internal reflection fluorescence microscopy. Biochim. Biophys. Acta Biomembr. 2016, 1858, 1244-1253.

(13) Dos Santos, M. C.; Déturche, R.; Vézy, C.; Jaffiol, R. Axial nanoscale localization by normalized total internal reflection fluorescence microscopy. Opt. Lett. 2014, 39, 869-872.

(14) Stabley, D. R.; Oh, T.; Simon, S. M.; Mattheyses, A. L.; Salaita, K. Real-time fluorescence imaging with $20 \mathrm{~nm}$ axial resolution. Nat. Commun. 2015, 6, 8307.

(15) Bourg, N.; Mayet, C.; Dupuis, G.; Barroca, T.; Bon, P.; Lécart, S.; Fort, E.; LévêqueFort, S. Direct optical nanoscopy with axially localized detection. Nat. Photonics 2015, 9, $587-593$.

(16) Deschamps, J.; Mund, M.; Ries, J. 3D superresolution microscopy by supercritical angle detection. Opt. Express 2015, 22, 29081-29091.

(17) Winterflood, C. M.; Ruckstuhl, T.; Verdes, D.; Seeger, S. Nanometer axial resolution by three-dimensional supercritical angle fluorescence microscopy. Phys. Rev. Lett. 2010, 105, 108103.

(18) Thevenaz, P.; Ruttimann, U. E.; Unser, M. A pyramid approach to subpixel registration based on intensity. IEEE Trans. Image Process. 1998, 7, 27-41. 
(19) Miura, K.; Rueden, C.; Hiner, M.; Schindelin, J.; Rietdorf, J. ImageJ Plugin CorrectBleach V2.0.2. Zenodo. https://doi.org/10.5281/zenodo.30769, 2014.

(20) Paszek, M. J.; DuFort, C. C.; Rubashkin, M. G.; Davidson, M. W.; Thorn, K. S.; Liphardt, J. T.; Weaver, V. M. Scanning angle interference microscopy reveals cell dynamics at the nanoscale. Nat. Methods 2012, 9, 825-827.

(21) Chizhik, A. I.; Rother, J.; Gregor, I.; Janshoff, A.; Enderlein, J. Metal-induced energy transfer for live cell nanoscopy. Nat. Photonics 2014, 8, 124-127.

(22) Isbaner, S.; Karedla, N.; Kaminska, I.; Ruhlandt, D.; Raab, M.; Bohlen, J.; Chizhik, A.; Gregor I.; Tinnefeld, P.; Enderlein, J.; Tsukanov, R. Axial colocalization of single molecules with nanometer accuracy using metal-induced energy transfer. Nano Lett. 2018, 18, 26162622.

(23) Arganda-Carreras, I.; Kaynig, V.; Rueden, C.; Eliceiri, K. W.; Schindelin, J.; Cardona, A.; Sebastian Seung, H. Trainable Weka Segmentation: a machine learning tool for microscopy pixel classification. Bioinformatics 2017, 33, 2424-2426. 